\title{
Síndrome de Guillain-Barré posterior a vacunación en lactante menor
}

\author{
Guillain-Barré syndrome after vaccination in an infant
}

\author{
Daniel Felipe Cepeda-Bareño', Josué Delgado-Serranoø
}

Fecha correspondencia:

Recibido: marzo 5 de 2020.

Revisado: julio 16 de 2020.

Aceptado: agosto 11 de 2020.

Forma de citar:

Cepeda-Bareño DF, Delgado-

Serrano J. Síndrome de Guillain-

Barré posterior a vacunación en

lactante menor. Rev CES Med

2020; 34(2): 162-168.

\section{Open access}

(C) Derecho de autor

Licencia creative commons

Ética de publicaciones

Revisión por pares

Gestión por Open Journal System

DOl: http://dx.doi.org/10.21615/

cesmedicina.34.2.8

ISSN 0120-8705

e-ISSN 2215-9177

Sobre los autores:

1. Médico, Hospital Local del

Norte, Bucaramanga, Santander,

Colombia.

2. Médico, Universidad Autónoma de Bucaramanga, Bucaramanga,

Santander, Colombia.

Comparte

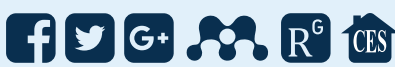

\section{Resumen}

El síndrome de Guillain-Barré es un trastorno autoinmune que se presenta como polineuropatía paralítica flácida rápidamente progresiva y puede ser fatal. Su diagnóstico es clínico y se apoya en la electromiografía. En la mayoría de los casos está precedido por un proceso infeccioso respiratorio o gastrointestinal, pero existen casos excepcionales en los que aparece posterior a vacunación, particularmente contra influenza. El objetivo de este artículo es presentar el caso de un lactante menor diagnosticado con síndrome de Guillain-Barré posterior a la aplicación de vacunas de los seis meses de edad. Es importante para el clínico considerar esta asociación al momento de definir diagnósticos diferenciales, dejando claro que el beneficio de la vacunación siempre superará los riesgos.

Palabras clave: Síndrome de Guillain-Barré; Vacunas contra la Influenza; Lactante; Paresia; Autoinmunidad; Pediatría.

\begin{abstract}
Guillain-Barré syndrome is potentially fatal autoimmune disorder that presents as rapidly progressive flaccid paralytic polyneuropathy. Diagnosis is based on clinical findings and supported by electromyography. In most cases, it is preceded by a respiratory or gastrointestinal infection, but exceptional cases in which it appears after vaccination have been reported. This article aims to present the case of a young infant diagnosed with Guillain-Barré syndrome after influenza vaccination. It is important for the clinician to consider this association when defining differential diagnoses when dealing with similar cases, stating that the benefits of vaccination clearly outweight its risks.
\end{abstract}

Keywords: Guillain-Barre Syndrome; Influenza Vaccines; Infant; Paresis; Autoinmmunity; Pediatrics.

\section{Introducción}

El síndrome de Guillain Barré es la causa más frecuente de parálisis flácida en la población pediátrica. Se trata de una condición autoinmune grave, potencialmente mortal, que se presenta como una polineuropatía paralítica, manifestada en forma de debilidad muscular simétrica y distal, con rápida progresión hacia el compromiso proximal y la arreflexia generalizada, con reportes de falla respiratoria hasta en el $20-30 \%$ de los casos $(1,2)$. 
En un $70 \%$ de los casos el síndrome de Guillain-Barré está precedido por una infección respiratoria o gastrointestinal dos a cuatro semanas antes de los síntomas neurológicos y menos del $10 \%$ posterior a vacunación.
Existen varios subtipos de esta condición, siendo la más frecuente (50 \% de los casos) la polineuropatía desmielinizante inflamatoria aguda. En las otras formas clínicas entre el 10 al $15 \%$ corresponde a variantes axonales como la neuropatía axonal motora aguda, la neuropatía axonal motora y sensitiva aguda y el síndrome de Miller-Fisher (oftalmoplejía, ataxia y arreflexia sin debilidad muscular) $(3,4)$.

Su diagnóstico es eminentemente clínico y los exámenes paraclínicos se utilizan para descartar infección en el sistema nervioso central, evaluando la relación albumina/celularidad en el líquido cefalorraquídeo, y, en el caso de la electromiografía y la velocidad de conducción, para confirmar el diagnóstico y la severidad del cuadro; sin embargo, algunos pacientes pueden tener electromiografía normal durante las dos primeras semanas de evolución (5).

En un $70 \%$ de los casos el síndrome está precedido por una infección respiratoria o gastrointestinal dos a cuatro semanas antes de los síntomas neurológicos (2) y menos del $10 \%$ posterior a vacunación (6). Aunque es muy debatido, se ha planteado relación causal y excepcionalmente se ha reportado su aparición luego de vacunación contra influenza, rabia y sarampión (7).

El objetivo de este reporte es presentar el caso de un lactante menor con diagnóstico de síndrome de Guillain-Barré posterior a la vacunación de los seis meses de edad, con el fin de concientizar al clínico en la posibilidad de existencia de esta asociación excepcional.

\section{Caso clínico}

Un lactante masculino de siete meses, sin antecedentes perinatales de importancia, previamente sano, fue llevado a la consulta por un cuadro clínico de una semana de evolución, caracterizado por fiebre cuantificada hasta 38,5 C y episodios eméticos ocasionales, manejado en casa con acetaminofén y suero oral, con mejoría parcial. Dos días antes del inicio de este cuadro clínico se le había realizado vacunación, correspondiente a los seis meses de vida según el esquema de Colombia (pentavalente, polio oral e influenza).

En la institución de primer nivel donde fue atendido originalmente se consideró cuadro viral agudo y dieron egreso con manejo sintomático. Tres días después de la primera consulta fue llevado nuevamente por aparición de debilidad muscular generalizada y persistencia de fiebre, tras lo cual dieron manejo con azitromicina oral de forma ambulatoria.

Al sexto día fue llevado al servicio de urgencias de un hospital de segundo nivel por presentar dificultad respiratoria, disfonía, tos seca y deposiciones líquidas con sangre macroscópica. Se decidió manejar la disentería con ceftriaxona y un presunto cuadro de crup con esteroide sistémico y nebulizaciones con adrenalina. Sin embargo, ante la presencia de síndrome de hipotonía muscular y la dificultad respiratoria asociada, se remitió a institución de mayor complejidad en donde se le realizaron paraclínicos que no fueron sugestivos de infección bacteriana concomitante (cuadro 1). 
Cuadro 1. Resultados de laboratorio

\begin{tabular}{|c|c|}
\hline Hemograma tipo IV & $\begin{array}{l}\text { Recuento de leucocitos: } 6,99 \times 10^{3} / \mathrm{uL} \\
\text { Neutrófilos: } 3,86 \times 10^{3} / \mathrm{uL} \\
\text { Linfocitos: } 2,49 \times 10^{3} / \mathrm{uL} \\
\text { Recuenta de glóbulos rojos: } 4,29 \times 10^{6} / \mathrm{u} \\
\text { Hemoglobina: } 11,4 \mathrm{~g} / \mathrm{dL} \\
\text { Hematocrito: } 34,5 \% \\
\text { Recuento de plaquetas: } 324 \times 10^{3} / \mathrm{uL}\end{array}$ \\
\hline Glucosa sérica & $99 \mathrm{mg} / \mathrm{dL}$ \\
\hline Hemocultivo No. 1 & Negativo a los cinco días de incubación \\
\hline Hemocultivo No. 2 & Negativo a los cinco días de incubación \\
\hline Urocultivo & Negativo a las 24 horas de incubación \\
\hline Gases arteriales & $\begin{array}{l}\mathrm{FlO}_{2}: 0,24 \\
\mathrm{Ph}: 7,38 \\
\mathrm{PCO}_{2}: 27 \mathrm{~mm} \mathrm{Hg} \\
\mathrm{PO}_{2}: 171 \mathrm{~mm} \mathrm{Hg} \\
\mathrm{CHCO}_{3}-: 15,9 \mathrm{mmol} / \mathrm{L} \\
\mathrm{SO}_{2}: 99 \% \\
\mathrm{PAFl}: 714 \mathrm{mmHg} \\
\text { Lactato: } 12,6 \mathrm{mg} / \mathrm{dL}(1,4 \mathrm{mmol} / \mathrm{L})\end{array}$ \\
\hline
\end{tabular}

La electromiografía con velocidades de conducción de las cuatro extremidades, fue compatible con polineuropatía pura, axonal y mielínica, predominio axonal, bilateral, de predominio en el hemicuerpo izquierdo, propio del síndrome de Guillain Barré, Hughes tipo IV.
Fue valorado por el servicio de Neurología Infantil que encontró arreflexia e hipotonía generalizada y del esfínter anal, percepción de estímulos nociceptivos únicamente en hemicuerpo derecho, sin alteración de la consciencia ni de los pares craneales. Se consideró cuadriparesia flácida con sospecha de polirradiculopatía tipo Guillain Barré, por lo que fue llevado a unidad de cuidados intensivos ante el riesgo inminente de falla ventilatoria. Aunque el paciente no presentaba signos ni síntomas de encefalitis ni meningitis, se intentó hacer punción lumbar, que fue fallida en tres intentos.

Posteriormente, se realizó electromiografía con velocidades de conducción de las cuatro extremidades, la cual fue compatible con presencia de polineuropatía motora pura, axonal y mielínica, predominio axonal, bilateral, de predominio en el hemicuerpo izquierdo, propio del síndrome de Guillain Barré, Hughes tipo IV (figura 1),

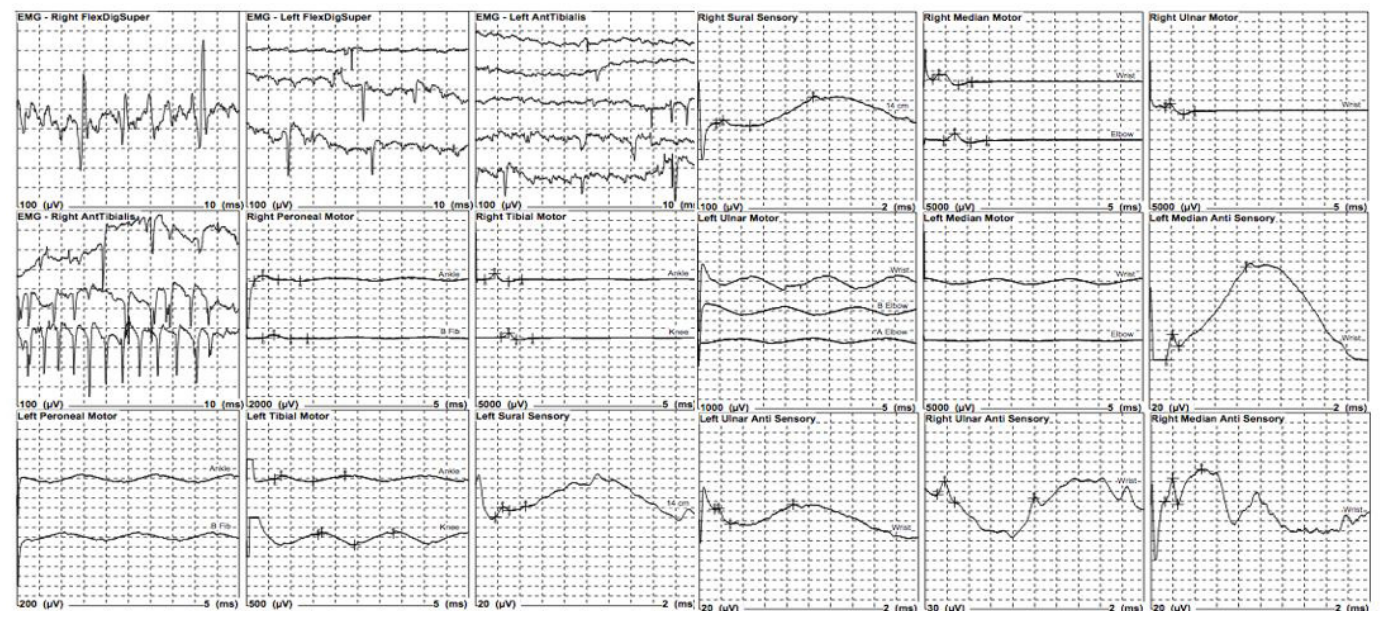

Figura 1. Electromiografía y velocidad de conducción de las cuatro extremidades 
El diagnóstico se basa en los hallazgos clínicos, en el antecedente de síntomas prodrómicos respiratorios o gastrointestinales, presentados dos a cuatro semanas antes del cuadro de debilidad muscular, así como en el hallazgo exámenes paraclínicos que no evidencien infección y en asociación a pruebas diagnósticas como la electromiografía y velocidad de conducción.
Durante su estancia en la unidad de cuidados intensivos no requirió soporte ventilatorio. Se manejó con inmunoglobulina IV durante tres días, tras lo cual se trasladó a cuidados intermedios, en donde se realizó terapia física. Cinco días después fue trasladado a unidad de cuidad básico, con mejoría progresiva del sostén cefálico y de la fuerza de miembros superiores e inferiores. Tras la adecuada evolución y la realización de cindeglución que permitió la autorización de dieta oral, fue dado de alta a los 14 días de hospitalización.

\section{Discusión}

El síndrome de Guillain-Barré es la polineuropatía aguda más frecuente en la población pediátrica en edades de uno a cinco años y es más frecuente en el sexo masculino (8); su incidencia anual estimada a nivel global es de 0,34 a 1,34 casos por cada 100 000 personas (9).

Su fisiopatología, que es de origen autoinmune, depende del subtipo: la polineuropatía desmielinizante inflamatoria aguda es la forma clínica más frecuente y se caracteriza por un proceso inflamatorio agudo a nivel de las raíces de nervios motores y sensitivos, secundario a la acción de anticuerpos contra las células de Schwann (3,4). En las formas axonales, los anticuerpos se dirigen contra los gangliósidos, entre los cuales el compromiso de GM1 y GD1a se relaciona con la expresión clínica de neuropatía axonal motora aguda y la afectación de GQ1B y GT1a con el síndrome de Miller-Fisher (10). Se cree que la generación de estos anticuerpos es por mimetismo molecular de algunos antígenos microbianos, más frecuentemente por Mycoplasma pneumoniae y Campylobacter jejuni (11).

El diagnóstico se basa en los hallazgos clínicos, en el antecedente de síntomas prodrómicos respiratorios o gastrointestinales, presentados dos a cuatro semanas antes del cuadro de debilidad muscular, así como en el hallazgo exámenes paraclínicos que no evidencien infección y en asociación a pruebas diagnósticas como la electromiografía y velocidad de conducción, las cuales confirman el diagnóstico $(1,2)$

Para el caso presentado, los datos del clínicos y de la electromiografía con velocidad de conducción, llevaron al diagnóstico de síndrome de Guillain Barré. A pesar de que la punción lumbar no fue posible, el paciente no presentó signos ni síntomas de neuroinfección, y mejoró con el manejo instaurado. Es importante mencionar que los síntomas gastrointestinales fueron concomitantes al proceso neurológico y que los síntomas respiratorios aparentes, que se presentaron pocos días antes, se pudieron deber al compromiso de la mecánica ventilatoria por causa neurológica.

Teniendo en cuenta lo anterior, se plantea la posibilidad de estar ante un caso de síndrome de Guillain-Barré posterior a vacunación, con mayor probabilidad de tratarse de una reacción secundaria a la vacuna contra el virus de la influenza, la cual ha sido reportada previamente (11-13). Otras vacunas que pueden causar el síndrome, como la rabia y el sarampión (7), no fueron aplicadas al paciente.

Una investigación canadiense, realizada a partir de 13 millones de pacientes vacunados contra la influenza entre 1993 y 2011, encontró un riesgo relativo de 1,52 (95\% Cl: 1,17-1,99) y una incidencia estimada de tres casos por cada millón de vacunas administradas (14). Igualmente, esta asociación se demostró en un estudio realizado en Estados Unidos entre 1992 a 1994 con un riesgo relativo de 1,7 (95\% Cl: 1,0-2,8) (15). 
Los beneficios de la vacunación superan ampliamente el riesgo de desarrollar síndrome de Guillain-Barré, por lo que nunca se debe considerar la no aplicación de las vacunas en la población pediátrica.
En relación con población infantil, hay pocos estudios: en Corea del Sur se documentan 14 casos de adolescentes con síndrome de Guillain-Barré luego de vacunación contra influenza (16); así como un reporte de caso de un paciente de 11 años, vacunado contra influenza dos semanas antes del diagnóstico de síndrome de Guillain Barré, quien, similar a lo ocurrido con el paciente descrito, presentó mejoría de los síntomas neurológicos tras la administración de inmunoglobulina intravenosa (17); sin embargo, no se dispone de cohortes en otros grupos poblacionales pediátricos, ni de reportes más recientes que planteen la sospecha de relación causal, la cual es excepcional y no debe de ninguna manera desincentivar la vacunación.

Independientemente de la causa, el manejo del síndrome es el mismo, definiendo, en primer lugar, la necesidad de asistencia respiratoria con ventilación mecánica, principalmente en pacientes con distrés respiratorio, hipotensión y resultado de electromiografía que reporte un puntaje Hughes mayor o igual de IV (18). En el caso descrito no se requirió soporte ventilatorio.

El tratamiento específico puede ser la inmunoglobulina intravenosa o la plasmaféresis; la primera ha demostrado reducción en la duración y la severidad de los síntomas, con mejores resultados en la neuropatía de tipo axonal motora aguda (19). Lo anterior se evidenció en el caso descrito, en el cual se demostró la presencia de una forma axonal con predominio motor y se logró recuperación del sostén cefálico y la motricidad de los miembros superiores en los primeros cinco días de la aplicación de inmunoglobulina, con recuperación progresiva y posterior egreso a los 14 días.

En conclusión, el síndrome de Guillain-Barré se puede presentar posterior a vacunación, más frecuentemente a la vacuna contra la influenza, y es un evento de presentación excepcional. Se resalta la importancia de sospechar este síndrome ante la presencia de síntomas de debilidad aguda, para realizar un diagnóstico y tratamiento oportunos, procurando revisar siempre los antecedentes de síntomas gastrointestinales o respiratorios, así como el esquema de vacunación reciente.

Por último, es importante resaltar que los beneficios de la vacunación superan ampliamente el riesgo de síndrome de Guillain-Barré, por lo cual nunca se debe considerar la no aplicación de las vacunas en la población pediátrica.

\section{Consideraciones éticas}

La consulta de la historia clínica completa, así como la elaboración del presente caso clínico, se hicieron tras la aprobación y firma de consentimiento informado de la madre del menor y de un tercero que actuó como testigo.

\section{Conflictos de interés}

Los autores declaran no tener conflictos de interés.

\section{Fuente de financiación}

Ninguna. 


\section{Bibliografía}

1. Willison HJ, Jacobs BC, Van Doorn PA. Guillain-Barré Syndrome. Lancet. 2016 Aug 13:388(10045):717-27. doi: 10.1016/s0140-6736(16)00339-1.

2. Ryan MM. Pediatric Guillain-Barré syndrome. Curr Opin Pediatr. 2013;25:689-93. doi: 10.1097/MOP.0b013e328365ad3f.

3. Karalok, ZS, Taskin BD, Yanginlar ZB, Gurkas E, Guven A, Degerliyurt A, Kose, G. Guillain-Barré syndrome in children: subtypes and outcome. Child's Nervous System. 2014;34(11):2291-2297. doi: 10.1007/s00381-018-3856-0.

4. Devos D, Magot A, Perrier-Boeswillwald J, Fayet G, Leclair- Visonneau L, Ollivier $Y$ et al. Guillain-Barré syndrome during childhood: particular clinical and electrophysiological features. Muscle Nerve. 2013;48:247-51. doi: 10.1002/mus.23749.

5. Top KA, Desai S, Moore D, Law BJ, Vaudry W, Halperin SA, et al. Guillain-Barré syndrome after immunization in Canadian children (1996-2012). Pediatr Infect Dis J. 2015;34:1411-3. doi: 10.1097/INF.0000000000000903.

6. Schessl J, Luther B, Kirschener J, Mauff G, Korinthenberg R. Infections and vaccinations preceding childhood Guillain-Barré symptom complex: a prospective study. Eur J Pediatr. 2006;165:605-12.

Una investigación canadiense, realizada a partir de 13 millones de pacientes vacunados contra la influenza entre 1993 y 2011, encontró un riesgo relativo de 1,52 (95\% Cl: 1,17-1,99) y una incidencia estimada de tres casos por cada millón de vacunas administradas.
7. Morgan L. The child with acute weakness. Clin Pediatr Emerg Med. 2015;16:1928. Doi: 10.1016/j.cpem.2015.02.004.

8. Yuki N, Hartung HP. Guillain-Barré syndrome. N Engl J Med 2012;366(24):2294304. doi: 10.1056/NEJMra1114525.

9. Sejvar JJ, Baughman AL, Wise M, Morgan OW. Population incidence of Guillain-Barré syndrome: a systematic review and meta-analysis. Neuroepidemiology. 2011;36(2):123-133. doi:10.1159/000324710.

10. Esposito S, Longo MR. Guillain-Barré syndrome. Autoimmun Rev. 2017 Jan;16(1):96-101. doi: 10.1016/j.autrev.2016.09.022.

11. Principi N, Esposito S. Vaccine-preventable diseases, vaccines and Guillain-Barre's Syndrome.Vaccine.2019Sep 3;3(37):5544-5550. doi:10.1016/j.vaccine.2018.05.119.

12. Sanz Fadrique R, Martín Arias L, Molina-Guarneros JA, Jimeno Bulnes N, García Ortega P. Guillain-Barré syndrome and influenza vaccines: current evidence. Rev Esp Quimioter. 2019;32(4):288-295.

13. Haber P, Moro PL, Cano M, Vellozzi C, Lewis P, Woo EJ et al. Post-Licensure surveillance of trivalent live-attenuated influenza vaccine in children aged 2-18 years, Vaccine Adverse Event Reporting System, United States, July 2005-June 2012. J Pediatric Infect Dis Soc. 2015;4(3):205-213. doi: 10.1093/jpids/piu034

14. Kwong JC, Vasa PP, Campitelli MA, Hawken S, Wilson K, Rosella LC, et al. Risk of Guillain-Barré syndrome after seasonal influenza vaccination and influenza health-care encounters: a self-controlled study. Lancet Infect Dis 2013;13:76976. doi: 10.1016/S1473-3099(13)70104-X. 
15. Uurlink DN, Stukel TA, Kwong J, Kopp A, McGeer A, Upshur RE, et al. GuillainBarre syndrome after influenza vaccination in adults-a population-based study. Arch Int Med 2006;166:2217-21.

16. Lee SJ, Kim YO, Woo YJ, Kim MK, Nam TS, Cho YK, et al. Neurologic adverse events following influenza $A(H 1 N 1)$ vaccinations in children. Pediatrics International. 2012;54(3):325-330. doi: 10.1111/j.1442-200X.2012.03568.x.

17. Tremblay ME., Closon A, D'Anjou G, Bussières JF. Guillain-Barré syndrome following $\mathrm{H} 1 \mathrm{~N} 1$ immunization in a pediatric patient. Annals of Pharmacotherapy 2010; 44(7-8), 1330-1333. doi: 10.1345/aph.1P078

18. Hu MH, Chen CM, Lin KL, Wang HS, Hsia SH, Chou ML, et al. Risk Factors of respiratory failure in children with Guilliain-Barré Syndrome. Pediatrics \& Neonatology. 2012;53(5):295-299. doi: https://doi.org/10.1016/j.pedneo.2012.07.003.

19. Kalita J, Kumar M, Misra UK. Role of IV immunoglobulin in indian children with Guillain Barré Syndrome. Pediatric Critical Care Medicine. 2019;20(7):652-659. doi: 10.1097/PCC.0000000000001935. 